\title{
Improved Lower Bounds for the Critical Probability of Oriented-Bond Percolation in Two Dimensions
}

\author{
V. Belitsky* \\ Universidade de São Paulo - Instituto de Matemática e Estatística \\ T. L. Ritchie ${ }^{\dagger}$ \\ Universidade de São Paulo - Instituto de Matemática e Estatística \\ Rua do Matão 1010, CEP 05508-900, São Paulo SP, Brazil.
}

\begin{abstract}
We present a coupled decreasing sequence of random walks on $\mathbb{Z}\left(\bar{X}^{(i)}, i \in \mathbb{N}\right)$ that dominates the edge process of oriented-bond percolation in two dimensions. Using the concept of "random walk in a strip ",we construct an algorithm that generates an increasing sequence of lower bounds that converges to the critical probability of oriented-bond percolation. Numerical calculations of the first ten lower bounds thereby generated lead to an improved,ie higher, rigorous lower bound to this critical probability, viz. $p_{c} \geq 0.63328$. Finally a computer simulation technique is presented; the use thereof establishes 0.64450 as a non-rigorous five-digit-precision (lower) estimate for $p_{c}$.
\end{abstract}

Key Words: oriented percolation, discrete time contact processes, critical probability, edge process, Markov chain in a strip, coupling, simulation .

\section{Introduction}

Oriented percolation in two dimensions or discrete time contact process on $\mathbb{Z}$ is a one-parameter family of discrete time stochastic processes defined on $\{0,1\}^{\mathbb{Z}}$; the parameter $p$, the infection rate, taking values on $[0,1]$. It is a well established fact that this family exhibits a fase transition, as the value of $p$ increases from 0 to 1: if $p<p_{c}{ }^{1}$, the process dies out almost surely; whereas if $p>p_{c}$, the process survives with positive probability (see [1] for instance).

As usual, in critical phenomena theory, an analytical expression for $p_{c}$ is unknown and its value has been estimated both in mathematical and physical literatures [1 Sec.6].

Up to the present moment, the best (rigorous) lower and upper bounds for $p_{c}$ are respectively 0.6298 and $2 / 3$ 2.

In this paper, we present an algorithm that generates an increasing sequence of lower bounds that converges to the critical probability of oriented bond percolation in two dimensions and, calculating the first ten lower bounds thereby generated, we were able to improve the best lower bound known up to now from 0.6298 to 0.63328 .

\footnotetext{
*Supported by CNPq (grant N.301637/91-1) / e-mail: belitsky@ime.usp.br

†Supported by a grant from CNPq / e-mail: thomasl@ime.usp.br

1 " $p_{c}$ " is known as the critical probability of the family.
} 
More specifically:

1. a numerical sequence of lower bounds for $p_{c},\left\{p_{c}^{(i)}\right\}_{i \in \mathbb{N}}$, was constructed in the following steps:

(a) Associated to a decreasing ${ }^{2}$ sequence(in $i$ ) of random walks on $\mathbb{Z},\left\{\bar{X}_{n}^{(i)}\right\}_{n \in \mathbb{N}}$, that dominate the edge process of oriented percolation, we constructed a sequence (in $i$ ) of finite, discrete time Markov chains, $\left\{Y_{n}^{(i)}\right\}_{n \in \mathbb{N}}$, whose transition probabilities are specific ${ }^{3}$ functions of $p$, the infection probability; and

(b) calculated the mathematical expectation of the random variable $\mathbb{E}\left(\bar{X}_{1}^{(i)}-\bar{X}_{0}^{(i)} \mid Y_{0}^{(i)}\right)$, the mean jump on the $Y$-configuration, with respect to $\pi^{(i)}$, the stationary measure for $Y_{\bullet}^{(i)}$.

(c) $p_{c}^{(i)}$ was then defined to be the (only) value of $p$ that nullifies the above expectation. In other words, when $p=p_{c}^{(i)}$, the random walk $\left\{\bar{X}_{n}^{(i)}\right\}_{n \in \mathbb{N}}$ has zero mean drift.

2. the numerical sequence $\left\{p_{c}^{(i)}\right\}_{i \in \mathbb{N}}$ was shown to converge in a non-decreasing fashion to $p_{c}$, ie $p_{c}^{(i)} \nearrow p_{c}$. Moreover,

3. the first ten lower bounds $\left(p_{c}^{(0)}, p_{c}^{(1)}, \ldots, p_{c}^{(9)}\right)$ were numerically calculated, thereby improving the best rigorous lower bound known up to the moment from 0.6298 to 0.63328 .

In the last section we present a simulation technique which in our opinion has important advantages in comparison with the usual so called Monte Carlo simulation techniques, in the sense that it exhibites a clear cut off between the subcritical and supercritical phases; thereby enabling a precise estimation of the critical probability of Oriented Bond Percolation without the aid of scaling techniques. By means thereof a lower bound for $p_{c}$ was obtained within a precision of 5 digits, viz $p_{c}^{1000}=0.64451$, so that we can (non-rigorously) state that $p_{c}=(0.64451 \pm 0.00001)$.

\section{Definitions and Constructions}

\subsection{The Enviroment}

Let $\mathcal{G} \equiv(\mathcal{V}, \mathcal{E})$ be the oriented graph, having $\mathcal{V}=\{(n, m): n \in \mathbb{N}$ and $(n+m)$ is even $\}$ as its set of vertices/sites, and $\mathcal{E}=\left\{e_{n m}^{l}, e_{n m}^{r}:(n, m) \in \mathcal{V}\right\}$ as its set of bonds.

Bond $e_{n, m}^{l}$ points from site $(n, m)$ to site $(n+1, m-1)$, whereas bond $e_{n, m}^{r}$ points from site $(n, m)$ to site $(n+1, m+1)$. Sometimes the natural association: $l \leftrightarrow-1 / r \leftrightarrow+1$ will be assumed through out this text.

It is useful to think of $n$ as a (discrete) time coordinate and of $m$ as a (discrete) space coordinate of the graph $\mathcal{G}$.

$\mathcal{V}_{n}$ denotes the $\mathrm{n}$-th slice of $\mathcal{V}$, ie $\mathcal{V}_{n}=\{(n, m) \in \mathcal{V}: n$ isfixed $\}$, and $\mathbb{Z}_{n}$ the set of integers $m$ such that $(m+n)$ is even.

It will be often useful in the forthcoming definitions to identify $\mathcal{V}_{n}$ as $\mathbb{Z}_{n}$, and to think of $\{0,1\}^{\mathcal{V}_{n}}$ as being $\{0,1\}^{\mathbb{Z}_{n}}$. Therefore arises the loose, yet natural, notation: $\eta_{n}(m) \equiv \eta_{n}(n, m), \eta_{n} \in\{0,1\}^{\mathcal{V}_{n}}$.

\footnotetext{
${ }^{2}$ in a sense to be specified later by means of coupling

${ }^{3}$ polinomial
} 


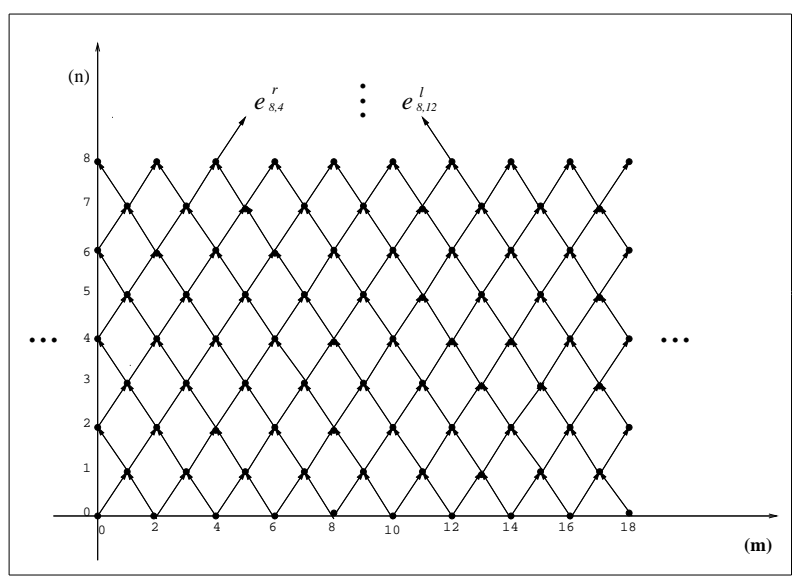

Figure 1: Graph $\mathcal{G}$, whereon the processes will be defined (Sec. 2.3 below).

\subsection{The Probability Structure}

Let $\left\{\xi_{n m}^{j}:(n, m) \in \mathcal{V}, j \in\{l, r\}\right\}$ be a family of independent and uniformly distributed ( onto $[0,1]$ ) random variables, defined on the same abstract probability space $\Omega$. Starting from this three-indexed countable family, we construct the four-indexed uncountable family of iid Bernoulli random variables on the same probability space $\Omega$ :

$$
\left\{p \xi_{n, m}^{j}:(n, m) \in \mathcal{V}, j \in\{l, r\}, p \in[0,1]\right\}
$$

such that

$$
{ }_{p} \xi_{n, m}^{j}=\mathbf{1}_{\left\{\xi_{n m}^{j} \leq p\right\}}
$$

It follows straightfowardly from (2) that

$$
\left\{\begin{array}{l}
\mathbb{P}\left(p \xi_{n m}^{j}=1\right)=p \\
\mathbb{P}\left(p \xi_{n m}^{j}=0\right)=1-p:=q \quad(b)
\end{array}\right.
$$

and the Fundamental Coupling:

$$
p_{1} \leq p_{2} \Rightarrow{ }_{p_{1}} \xi_{n m}^{j}(\omega) \leq p_{2} \xi_{n m}^{j}(\omega), \forall \omega \in \Omega,(n, m) \in \mathcal{V}, j \in\{l, r\}
$$

We observe that ${ }_{p} \xi_{n m}^{l}=1$ is interpreted as a channel open to infection propagation from site $(n, m)$ to site $(n+1, m-1) ;{ }_{p} \xi_{n m}^{r}=0$, as a channel obstructed to infection propagation from site $(n, m)$ to site $(n+1, m+1) ;$ and so forth $\ldots$

\subsection{The Processes}

\subsubsection{The Strengthened Discrete Time Contact Processes (SDTCP)}

$\eta_{n} \in\{0,1\}^{\mathcal{V}_{n}}$, is interpreted as a infection state on slice $\mathcal{V}_{n}$ :

$$
\left\{\begin{array}{l}
\eta_{n}(m)=1: \text { site } m \text { is infected at time } n \\
\eta_{n}(m)=0 ; \text { site } m \text { is healthy at time } n
\end{array}\right.
$$


Particulary $\eta_{0} \in\{0,1\}^{\mathcal{V}_{0}}$ will denote an initial state of infection over $\mathcal{V}_{0}$; the set of even integers, according to Section 2.1 above.

At this point, the following notation (to be used throughout this paper) should be kept into account: $\mathbb{N}=\{0,1,2, \cdots\}$ and $\overline{\mathbb{N}}=\mathbb{N} \cup\{\infty\}$.

Now for each $i \in \overline{\mathbb{N}}$, we define (by induction on $n$ ) the sequence of $\{0,1\}^{\mathcal{V}_{n}}$-valued random variables $\left\{{ }_{p} X_{n}^{(i)}\right\}_{n \in \mathbb{N}}$ as

\section{Definition 2.1.}

(a) ${ }_{p} X_{0}^{(i)}:=\eta_{0}$, so that ${ }_{p} X_{0}^{(i)}(m)=\eta_{0}(m), \forall m \in \mathbb{Z}_{0}$

(b) ${ }_{p} X_{n+1}^{(i)}(m)=\sup \left\{{ }_{p} X_{n}^{(i)}(m-1) \cdot{ }_{p} \xi_{n, m-1}^{r} ;{ }_{p} X_{n}^{(i)}(m+1) \cdot{ }_{p} \xi_{n, m+1}^{l} ; \mathbf{1}_{\left\{{ }_{p} \bar{X}_{n+1}^{(i)}-m>2 i\right\}}\right\}$,

where ${ }_{p} \bar{X}_{n+1}^{(i)}=\sup _{m \in \mathbb{Z}_{n} ; j \in\{-1,+1\}}\left\{(m+j):{ }_{p} X_{n}^{(i)}(m) \cdot \xi_{n m}^{j}=1\right\}$

The role of the indicator function in Definition [2.1 (b) above is to infect by force all the sites lying farther than $2 i$ on the left side of ${ }_{p} \bar{X}_{n+1}^{(i)}$, the utmost right infected site at time $n+1$. It is natural,therefore, to call the stochatic process $\left\{{ }_{p} X_{n}^{(i)}\right\}_{n \in \mathbb{N}}$, defined above, as the Strengthened Discrete Time Contact Process (of i-th order and infection parameter $p$ ).

It follows directly from Definition 2.1] that

(a) when $i=\infty$, the indicator function does not act anymore, and we recover the ordinary Discrete Time Contact Process, also called Oriented Percolation in Two Dimensions, described in [1. Sec. 2]. So forth in this paper we shall refer to it as $\left\{{ }_{p} X_{n}^{(\infty)}\right\}_{n \in \mathbb{N}}$;

(b) the sequence of SDTCPs $\left\{{ }_{p} X_{n}^{(i)}\right\}_{n \in \mathbb{N}}$ is decreasing in $i$, in the following sense:

$$
i \leq j ; i, j \in \overline{\mathbb{N}} \Rightarrow{ }_{p} X_{n}^{(i)}(m)[\omega] \geq{ }_{p} X_{n}^{(j)}(m)[\omega], \forall \omega \in \Omega,(n, m) \in \mathcal{V}, p \in[0,1]
$$

In particular, the Oriented Percolation Process is the weakest of them all.

Inequality [6 above is called Coupling of First Kind

(c) the family of SDTCPs $\left\{{ }_{p} X_{n}^{(i)}\right\}_{n \in \mathbb{N}}$ is increasing in $p$ in the following sense

$$
p_{1} \leq p_{2} \Rightarrow{ }_{p_{1}} X_{n}^{(i)}(m)[\omega] \leq{ }_{p_{2}} X_{n}^{(i)}(m)[\omega], \forall \omega \in \Omega,(n, m) \in \mathcal{V}, i \in \overline{\mathbb{N}}
$$

Inequality 7 above is called Coupling of Second Kind

In this paper, unless otherwise stated, $\eta_{0}$ will be chosen to be $\mathbf{1}_{\bullet} \leq 0 \in\{0,1\}^{\mathcal{V}_{0}}$, where

$$
\mathbf{1}_{\bullet} \leq 0(m)=\left\{\begin{array}{l}
1, m \leq 0 \\
0, m>0
\end{array}\right.
$$

We shall write $\left\{{ }_{p}^{\eta} X_{n}^{(i)}\right\}_{n \in \mathbb{N}}$ to emphasize that $\eta_{0}=\eta$, when $\eta$ is some specific element of $\{0,1\}^{\mathcal{V}_{0}}$ ( probably different from $\mathbf{1} \leq 0)$. Therefore the symbols $\left\{{ }_{p} X_{n}^{(i)}\right\}_{n \in \mathbb{N}},\left\{{ }_{p}^{\mathbf{1}}{ }_{p} X_{n}^{(i)}\right\}_{n \in \mathbb{N}},{ }^{\mathbf{1}}{ }_{p} X_{\bullet}(i),{ }_{p} X{ }_{\bullet}^{(i)}$ have all the same meaning and, for simplicity's sake, the last will be chosen, when no confusion may arise.

As usual in Particle System's notation we write $\eta_{n} \geq \theta_{n}$, whenever $\eta_{n}(m) \geq \theta_{n}(m)$ for every $m \in \mathbb{Z}_{n}$. Definition 2.1 also implies that, for $\eta, \theta \in\{0,1\}^{\mathcal{V}_{0}}$

$$
\eta \geq \theta \Rightarrow{ }_{p}^{\eta} X_{n}^{(i)}(m)[\omega] \geq{ }_{p}^{\theta} X_{n}^{(i)}(m)[\omega], \forall p \in[0,1],(n, m) \in \mathcal{V}, \omega \in \Omega, i \in \overline{\mathbb{N}}
$$

Inequality 9 is called Coupling of Third Kind. 


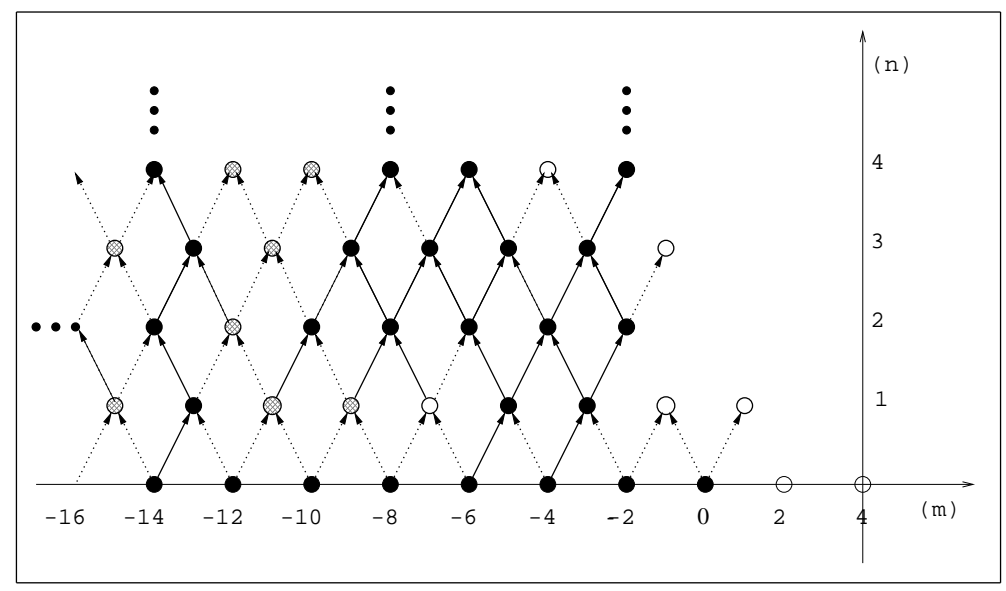

Figure 2: A realization $\omega$ of the ${ }_{p} X_{\bullet}^{(2)}$ process . Full-black arrows are open, whereas dotted ones are closed to infection propagation. Black sites/cells are infected, white ones are healthy, while the gridded ones were reinfected.

\subsubsection{The Right Edge Processes (REP)}

Given a particular SDTCP ${ }_{p} X_{\bullet}^{(i)}$ and assuming that ${ }_{p} \bar{X}_{0}^{(i)} \stackrel{\text { def }}{=} 0$, the second line of Definition [2.1(b) above defines a (non-markovian) random process on $\mathbb{Z}$ denoted by $\left\{{ }_{p} \bar{X}_{n}^{(i)}\right\}_{n \in \mathbb{N}}\left(\right.$ or ${ }_{p} \bar{X}_{\bullet}^{(i)}$ in abbreviated fashion). In Figure 2 above ${ }_{p} \bar{X}_{0}^{(2)}=0,{ }_{p} \bar{X}_{1}^{(2)}=-3,{ }_{p} \bar{X}_{2}^{(2)}=-2,{ }_{p} \bar{X}_{3}^{(2)}=-3,{ }_{p} \bar{X}_{4}^{(2)}=-2, \ldots$

Again, in case of $i=\infty$, the Edge Process of Oriented Percolation cited in [1] is recovered. Throughout this text, this term will be employed in a generilized way $(i \in \overline{\mathbb{N}})$.

It is useful to think of ${ }_{p} \bar{X}_{\bullet}^{(i)}$ as random walks on $\mathbb{Z}$.

\subsubsection{The Induced Markov Chains}

Definition 2.2 The Markov chain $\left\{{ }_{p} Y_{n}^{(i)}\right\}_{n \in \mathbb{N}}, i \in \mathbb{N}$, with state space $\mathcal{S}^{(i)}=\{0,1\}^{\{2,4, \ldots, 2 i\}}$ defined by

$$
{ }_{p} Y_{n}^{(i)}(2 j)={ }_{p} X_{n}^{(i)}\left({ }_{p} \bar{X}_{n}^{(i)}-2 j\right), 1 \leq j \leq i
$$

is called Induced Markov Chain(IMC) for the SDTCP ${ }_{p} X_{\bullet}^{(i)}$.

In what follows, a generic element $\sigma \in \mathcal{S}^{(i)}$, will be labeled by $n \in\left\{0,1, \ldots, 2^{i}-1\right\}$ according to

$$
\sigma=\sigma_{n} \Leftrightarrow n=\sum_{j=1}^{i} 2^{i-j} \sigma(2 j)
$$

Accordingly, in Figure 2 above ${ }_{p} Y_{0}^{(2)}=\sigma_{3},{ }_{p} Y_{1}^{(2)}=\sigma_{2},{ }_{p} Y_{2}^{(2)}=\sigma_{3},{ }_{p} Y_{3}^{(2)}=\sigma_{3},{ }_{p} Y_{4}^{(2)}=\sigma_{1}, \ldots$ The transition probabilities for the IMC ${ }_{p} Y_{\bullet}^{(i)}$

$$
q_{l m}^{(i)}=\mathbb{P}\left[{ }_{p} Y_{n+1}^{(i)}=\sigma_{m} \mid{ }_{p} Y_{n}^{(i)}=\sigma_{l}\right], 0 \leq l, m \leq 2^{i}-1
$$


are all strictly positive polinomial functions of $p$ (provided $0<p<1$ ). Therefore $\pi^{(i)}$, its stationary measure on $\mathcal{S}^{(i)}$, is well defined.

The notation

$$
\pi_{l}^{(i)} \stackrel{\text { def }}{=} \pi^{(i)}\left(\sigma_{l}\right), l=0,1, \ldots, 2^{i}-1
$$

is self explanatory.

At this point, in accoradance with [3] Sec.3.1], we state

\section{Definition 2.3}

$$
P_{(l, m, k)}^{(i)}(p) \stackrel{\text { def }}{=} \mathbb{P}\left[{ }_{p} \bar{X}_{n+1}^{(i)}={ }_{p} \bar{X}_{n}^{(i)}+(1-2 k),{ }_{p} Y_{n+1}^{(i)}=\sigma_{m} \mid{ }_{p} Y_{n}^{(i)}=\sigma_{l}\right], k \in \mathbb{N}, 0 \leq l, m \leq 2^{i}-1
$$

the transition probabilities from state $\sigma_{l}$ to state $\sigma_{m}$ with a jump of magnitude $(1-2 k)$

\section{Definition 2.4}

$$
M_{l}^{(i)}(p) \stackrel{\text { def }}{=} \sum_{k=0}^{\infty} \sum_{m=0}^{2^{i}-1}(1-2 k) \cdot P_{(l, m, k)}^{(i)}(p), \quad 0 \leq l \leq 2^{i}-1
$$

the mean jump/drift of the REP on configuration $\sigma_{l}$

and

\section{Definition 2.5}

$$
M^{(i)}(p) \stackrel{\text { def }}{=} \sum_{l=0}^{2^{i}-1} M_{l}^{(i)}(p) \cdot \pi_{l}^{(i)}
$$

the mean jump/drift of the REP.

$M^{(i)}(p)$ is also called the (right)edge speed in accordance with Section 2.4 bellow. As it will soon become clear, Definition [2.5] is of fundamental importance in this paper.

Under this framework, the SDTCPs ${ }_{p} X_{\bullet}^{(i)}$, described above, can be regarded, in case of $i \in \mathbb{N}$, as Markov Chains/Random Walks in a $2^{i}$-rowed Strip:

$$
{ }_{p} X_{n}^{(i)}={ }_{p} X_{n}^{(i)}\left({ }_{p} \bar{X}_{n}^{(i)},{ }_{p} Y_{n}^{(i)}\right)
$$

a slightly different idea of Markov Chains in a Half Strip, developed in [3] Sec.3.1].

\subsection{Critical Probabilities}

In Section 3 below it will be shown that, for $i \in \mathbb{N}$ and $p \in[0,1]$ :

(i) $M^{(i)}(p)$ is a scrictly incresing function of $p$;

(ii) $M^{(i)}(p)$ has only one real root ( in $\left.(0,1]\right)$;

(iii) $\frac{\bar{X}_{n}^{(i)}}{n} \stackrel{(n)}{\longrightarrow} M^{(i)}(p) \quad$ as 
In case of $i=\infty, \alpha(p)$, the (right) edge speed of oriented percolation, plays precisely the same role of $M^{(i)}(p)$ in the finite case depicted above ${ }^{4}$.(For details, see [1], for instance).So the notation

$$
M^{(\infty)}(p) \stackrel{\text { def }}{=} \alpha(p)
$$

suggests itself and we state the following

Definition 2.6 The critical (infection) probability $p_{c}^{(i)}$ for the family of stochastic processes ${ }_{p} X_{\bullet}^{(i)}, i \in \overline{\mathbb{N}}$, is the only real root of $M^{(i)}(p)$ (in $\left.(0,1]\right)$, the edge speed. Hence

$$
M^{(i)}\left(p_{c}^{(i)}\right)=0, \forall i \in \overline{\mathbb{N}}
$$

The heuristic meaning of Definition [2.6] above is :

(i) for $p<p_{c}^{(i)}, \lim _{n \rightarrow \infty}{ }_{p} \bar{X}_{n}^{(i)}=-\infty \quad a s$, ie the infection dies out with probability one;

(ii) for $p>p_{c}^{(i)}, \lim _{n \rightarrow \infty} \bar{X}_{n}^{(i)}=+\infty \quad a s$, ie the infection spreads out over all $\mathbb{Z}$.

In the sequel, we prove an important relation concerning the $p_{c}^{(i)} \mathrm{s}, i \in \overline{\mathbb{N}}$, just defined, viz. $p_{c}^{(i)} \nearrow_{i} p_{c}^{(\infty)} 5$. This non-decreasing convergence to the critical probability of Oriented Percolation (to be called The Convergence Theorem), besides the possibility of calculating the $p_{c}^{(i)} \mathrm{s}(i \in \mathbb{N})$ by algebraical means ( Section 4 below), is the cornerstone of this work.

\section{The Convergence Theorem and Preliminary Results}

Lemma $3.1 \frac{\bar{X}_{n}^{(i)}}{n} \stackrel{(n)}{\longrightarrow} M^{(i)}(p) \quad$ a s, $\forall i \in \overline{\mathbb{N}}, p \in(0,1]$

Proof:

First case $(i \in \mathbb{N})$ : Let $n_{l}^{(i)}$ be the (random) number of visits that the IMC ${ }_{p} Y_{\bullet}^{(i)}$ makes to state $\sigma_{l}$ up to time $n$ ( so that $\sum_{l=0}^{2^{i}-1} n_{l}^{(i)}=n$ ) and $J_{l, k}^{(i)}$ the $k^{\text {th }}$ jump of the REP ${ }_{p} \bar{X}_{\bullet}^{(i)}$ on state/row $\sigma_{l}$.

The (strong) Markov property of SDTCP ${ }_{p} X_{\bullet}^{(i)}$ makes $J_{l, k}^{(i)},: k \in\{1,2,3, \ldots\}$ iid rvs with $\mathbb{E}\left[J_{l, k}^{(i)}\right]=M_{l}^{(i)}$. Now

$$
\begin{array}{r}
\frac{p \bar{X}_{n}^{(i)}}{n}=\sum_{l=0}^{2^{i}-1} \sum_{k=1}^{n_{l}^{(i)}} \frac{J_{l, k}^{(i)}}{n}=\sum_{l=0}^{2^{i}-1} \sum_{k=1}^{n_{l}^{(i)}} \frac{J_{l, k}^{(i)}}{n_{l}^{(i)}} \cdot \frac{n_{l}^{(i)}}{n}=\sum_{l=0}^{2^{i}-1} \frac{n_{l}^{(i)}}{n} \sum_{k=1}^{n_{l}^{(i)}} \frac{J_{l, k}^{(i)}}{n_{l}^{(i)}}, \\
\frac{n_{l}^{(i)}}{n} \stackrel{(n)}{\longrightarrow} \pi_{l}^{(i)} \text { as and } \sum_{k=1}^{n_{l}^{(i)}} \frac{J_{l, k}^{(i)}}{n_{l}^{(i)}} \stackrel{n_{l}^{(i)}}{\longrightarrow} M_{l}^{(i)}(p) a s
\end{array}
$$

Taking the limit $n \rightarrow \infty$ and bearing definition 2.5 in mind, we get the desired result.

Second case $(i=\infty)$ : See [1 pag.1004]

${ }^{4} \alpha(p)=-\infty$, when $p<p_{c}$; so that the strict increasing behaviour does not apply to $\alpha(p)$ precisely.

${ }^{5} p_{c}^{(\infty)} \stackrel{\text { def }}{=} p_{c}$, putting the notations of Sections 1 and 2 into agreement. 
Lemma 3.2 For $i \in \mathbb{N}$, each function $M^{(i)}:(0,1] \rightarrow(-\infty, 1]$ is strictly increasing (in $\left.p\right)$. Moreover,

$$
p \mapsto M^{(i)}(p)
$$

$M^{(i)}$ is a surjection from $(0,1]$ onto $(-\infty, 1]$.

Idea of Proof:

The non-decreasing behaviour of $M^{(i)}(p)$ follows from the Second Kind of Coupling (inequality 7 above) and Lemma 3.1 just established. Definitions [2.2 2.3 2.4 and 2.5] make $M^{(i)}(p)$ a rational function of $p$ such that $M^{(i)}\left(0_{-}\right)=-\infty$ and $M^{(i)}(1)=(1)$; so $M^{(i)}(p)$ can not be constant on any interval $\left[p_{1}, p_{2}\right] \subset(0,1]$ and the strict behaviour follows.

\section{Commentary on Lemma 3.2 :}

At this point,it is worth observing that the function $\alpha(p) \stackrel{\text { def }}{=} M^{(\infty)}(p)$ is non-decreasing; strictly positive, when $p>p^{c}$; null, when $p=p_{c}$ and infinetly negative, when $p<p_{c}$. Again, the reference is [1].

Lemma 3.2 above yields

Corollary 3.3 For each $i \in \overline{\mathbb{N}}, M^{(i)}$ has only one real root in $(0,1]$, denoted by $p_{c}^{(i)}$, in accordace with Section 2.4.

Lemma 3.4 The sequence of functions $\left\{M^{(i)}(p)\right\}_{i \in \overline{\mathbb{N}}}$, is non increasing, ie $i \leq j ; i, j \in \overline{\mathbb{N}} \Rightarrow M^{(i)}(p) \geq M^{(j)}(p), \forall p \in(0,1]$. In particular $\alpha(p) \leq M^{(i)}(p) ; \forall i \in \mathbb{N}, \forall p \in(0,1]$.

Idea of Proof:

The non-increasing behaviour (in $i$ ) of $\left\{M^{(i)}(p)\right\}$ follows from the First Kind of Coupling ( inequality [ 6 above) and again from Lemma 3.1

Lemma 3.4 yields

Corollary 3.5 The numerical sequence $\left\{p_{c}^{(i)}\right\}_{i \in \mathbb{N}}$ is non-decreasing. Hence $\lim _{i \rightarrow \infty} p_{c}^{(i)}$ is well defined and $p_{c}^{(i)} \nearrow_{i} \lim _{i \rightarrow \infty} p_{c}^{(i)}$. Moreover $p_{c} \stackrel{\text { def }}{=} p_{c}^{(\infty)} \geq p_{c}^{(i)}, \forall i \in \mathbb{N}$

and

Corollary $3.6 p_{c}^{(\infty)} \geq \lim _{i \rightarrow \infty} p_{c}^{(i)}$.

Now we turn our attention to the reverse (and more difficult) inequality, viz. $p_{c} \leq \lim _{i \rightarrow \infty} p_{c}^{(i)}$. For that consider the following probability spaces:

- $(\Omega, \mathbb{P})$ : the abstract probability space, where the iid rvs $\xi_{n m}^{j}$ were defined;

- $\left(\mathcal{S}^{(i)}, \pi^{(i)}\right)$ : the finite probability space of the IMC $Y_{\bullet}^{(i)}$, endowed with its stationary measure $\pi^{(i)}$;

- $\left(\Omega \times \mathcal{S}^{(i)}, \mathbb{P} \times \pi^{(i)}\right)$ : the product space. 
In the product space, we define the stochastic processes :

- $\left\{{ }_{p} \overline{\mathcal{X}}_{n}^{(i)}\right\}_{n \in \mathbb{N}}$, by ${ }_{p} \overline{\mathcal{X}}_{n}^{(i)}((\omega, \sigma)) \stackrel{\text { def }}{=} \bar{X}_{n}^{(i)}(\omega), \forall(\omega . \sigma) \in \Omega \times \mathcal{S}^{(i)}$

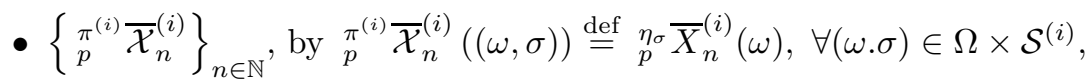

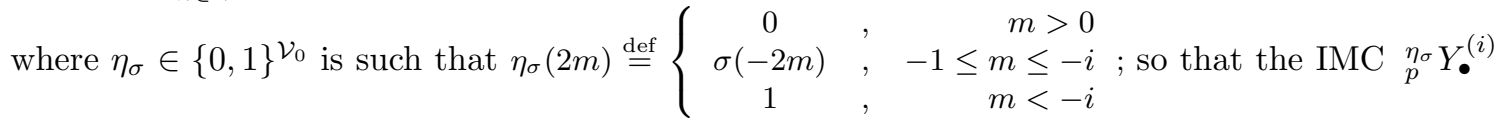
starts on state $\sigma \in \mathcal{S}^{(i)}$.

Now we state

Lemma 3.7 $\mathbb{E}\left[\frac{{ }_{p} \bar{X}_{n}^{(i)}}{n}\right] \geq M^{(i)}(p), \forall n, i \in \mathbb{N}, p \in(0,1]$.

Proof:

Inequality 9 implies that $\forall(\omega, \sigma) \in \Omega \times \mathcal{S}^{(i)},{ }_{p} \overline{\mathcal{X}}_{n}^{(i)}((\omega, \sigma))={ }_{p} \bar{X}_{n}^{(i)}(\omega) \geq{ }_{p}^{\eta_{\sigma}} \bar{X}_{n}^{(i)}(\omega)={ }_{p}^{\pi^{(i)}} \overline{\mathcal{X}}_{n}^{(i)}((\omega, \sigma))$. Integrating ( with respect to $\mathbb{P} \times \pi^{(i)}$ ) both sides of the inequality above, yields:

$$
\mathbb{E}\left[{ }_{p} \overline{\mathcal{X}}_{n}^{(i)}\right]=\left(\mathbb{E}_{\mathbb{P}}\left[{ }_{p} \bar{X}_{n}^{(i)}\right]\right) \geq \mathbb{E}\left[\begin{array}{l}
\pi_{p}^{(i)} \\
\mathcal{X}_{n}^{(i)}
\end{array}\right]\left(=n M^{(i)}(p)\right),
$$

where the last equality above comes from the fact that the process $\left\{\pi^{(i)} \overline{\mathcal{X}}_{n}^{(i)}\right\}_{n \in \mathbb{N}}$ has stationary increments of mean $M^{(i)}(p)$.

Figure 3 below refers to the next lemma :

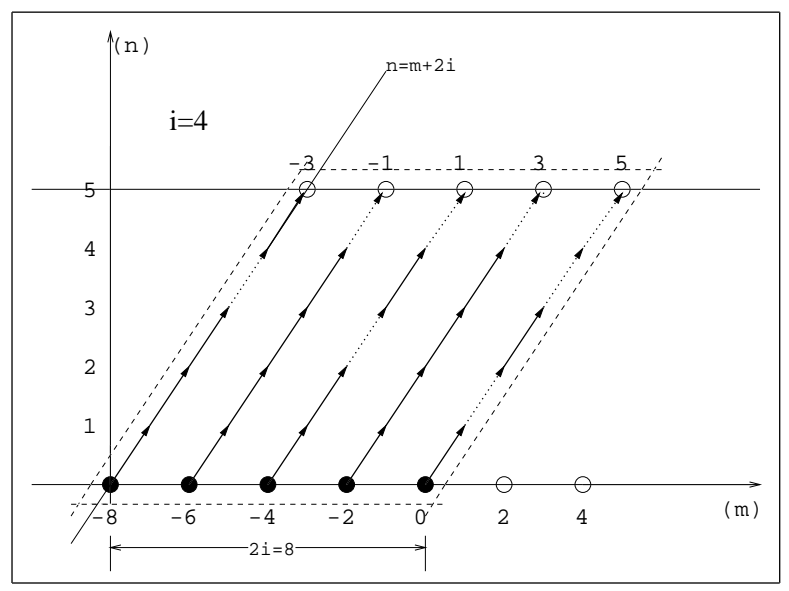

Figure 3: No reinfection can take place in the region inside the dashed parallelogram. The processes ${ }_{p} X_{\bullet}^{(4)}$ and ${ }_{p} X_{\bullet}^{(\infty)}$ are indistinguishable there.

Lemma 3.8 $\lim _{i \rightarrow \infty} \mathbb{E}\left[{ }_{p} \bar{X}_{n}^{(i)}\right]=\mathbb{E}\left[{ }_{p} \bar{X}_{n}^{(\infty)}\right]$ for all (fixed) $n \in \mathbb{N}$ and $p \in(0,1]$. 
Proof :

As the jumps to the right are bounded by +1 , it follows that $\forall \omega \in \Omega,{ }_{p} \bar{X}_{n}^{(i)} \leq n \Longrightarrow{ }_{p} \bar{X}_{n}^{(i)}-m \leq n-m$. However, for a site $(n, m)$ to be infected by force, we must have ${ }_{p} \bar{X}_{n}^{(i)}-m>2 i$ (Def. 2.1] (b) above). Hence, if $n-m \leq 2 i$, then ${ }_{p} \bar{X}_{n}^{(i)}-m \leq 2 i, \forall \omega \in \Omega$ and site $(\mathrm{n}, \mathrm{m})$ will not be infected by force, for any $\omega \in \Omega$. Accordingly, it can be proved by induction on $n$ that " $n-m \leq 2 i \Longrightarrow X_{n}^{(\infty)}(m)[\omega]=X_{n}^{(i)}(m)[\omega], \forall \omega \in \Omega$ ", ie all sites $(n, m)$ such that $n-m \leq 2 i$ have the same infection state regarding the processes ${ }_{p} X_{\bullet}^{(\infty)}$ and ${ }_{p} X_{\bullet}^{(i)}$. ( See Figure 3 above for the case: $i=4$.)

Hence, for any (fixed) time $n$,

$$
{ }_{p} \bar{X}_{n}^{(\infty)}<{ }_{p} \bar{X}_{n}^{(i)} \Longrightarrow{ }_{p} X_{n}^{(\infty)}(m)=0, \forall m \geq n-2 i
$$

The reasoning behind (13) is as follows:

$$
\begin{gathered}
\bar{p}_{p}^{(i)} \geq n-2 i \Longrightarrow{ }_{p} X_{n}^{(\infty)}\left({ }_{p} \bar{X}_{n}^{(i)}\right)={ }_{p} X_{n}^{(i)}\left({ }_{p} \bar{X}_{n}^{(i)}\right) \stackrel{\text { def }}{=} 1 \stackrel{\text { (囵) }}{\Longrightarrow} \\
{ }_{p} \bar{X}_{n}^{(i)} \stackrel{\text { def }}{=} \sup \left\{m \in \mathbb{Z}_{n}:{ }_{p} X_{n}^{(i)}(m)=1\right\}=\sup \left\{m \in \mathbb{Z}_{n}:{ }_{p} X_{n}^{(\infty)}(m)=1\right\} \stackrel{\text { def }}{=} \bar{X}_{p}^{(\infty)} \Longrightarrow \\
\left\{{ }_{p} \bar{X}_{n}^{(i)} \geq n-2 i\right\} \subset\left\{{ }_{p} \bar{X}_{n}^{(i)}={ }_{p} \bar{X}_{n}^{(\infty)}\right\} \Longrightarrow\left\{{ }_{p} \bar{X}_{n}^{(i)} \neq{ }_{p} \bar{X}_{n}^{(\infty)}\right\} \subset\left\{{ }_{p} \bar{X}_{n}^{(i)}<n-2 i\right\} \subset\left\{{ }_{p} \bar{X}_{n}^{(\infty)}<n-2 i\right\} \\
\text { ( 13) follows identifying }\left\{{ }_{p} \bar{X}_{n}^{(i)} \neq{ }_{p} \bar{X}_{n}^{(\infty)}\right\} \text { with }\left\{{ }_{p} \bar{X}_{n}^{(i)}>{ }_{p} \bar{X}_{n}^{(\infty)}\right\} \text { and }\left\{{ }_{p} \bar{X}_{n}^{(\infty)}<n-2 i\right\} \text { with }
\end{gathered}
$$
$\left\{{ }_{p} X_{n}^{(\infty)}(m)=0, \forall m \geq n-2 i\right\}$.

Now, observe that, if the infection is not present on the set $\left\{(n, m) \in \mathcal{V}_{n}: m \geq n-2 i\right\}$, all the paths joining its sites to slice $\mathcal{V}_{0}$ must be obstructed somewhere. In particular all the $(i+1)$ straight lines joining site $(0,-2 j)$ to site $(n, n-2 j), 0 \leq j \leq i$ must be interupted at some point (Figure 3 above). As these lines are made of different, independent bonds, the probability of this event equals $\left(1-p^{n}\right)^{i+1}$, and we have

$$
\mathbb{P}\left({ }_{p} \bar{X}_{n}^{(i)} \neq{ }_{p} \bar{X}_{n}^{(\infty)}\right) \leq \mathbb{P}\left({ }_{p} X_{n}^{(\infty)}(m)=0, \forall m \geq n-2 i\right) \leq\left(1-p^{n}\right)^{i+1}
$$

So that ${ }_{p} \bar{X}_{n}^{(i)} \stackrel{i}{\longrightarrow}{ }_{p} \bar{X}_{n}^{(\infty)}$ in probability, and there is a sub-sequence $\left(i_{k}\right)_{k \in \mathbb{N}}$ such that ${ }_{p} \bar{X}_{n}^{\left(i_{k}\right)} \stackrel{k}{\longrightarrow}{ }_{p} \bar{X}_{n}^{(\infty)} a s$ 4 Theorem 7.6]. As the whole sequence $\left\{{ }_{p} \bar{X}_{n}^{(i)}\right\}_{i \in \mathbb{N}}$ is non increasing (in i), we must have ${ }_{p} \bar{X}_{n}^{(i)} \stackrel{i}{\longrightarrow} \bar{X}_{n}^{(\infty)} a s$ as well, and we can apply the Monotone Convergence Theorem to conclude that $\mathbb{E}\left[{ }_{p} \bar{X}_{n}^{(i)}\right] \searrow^{i} \mathbb{E}\left[{ }_{p} \bar{X}_{n}^{(\infty)}\right], \forall n \in \mathbb{N}$.

Now we can prove the Convergence Theorem:

Theorem $3.9 p_{c}^{(i)} \nearrow_{i} p_{c}$

Proof : Suppose that $\lim _{i \rightarrow \infty} p_{c}^{(i)}<p_{c}$, so that we can choose $p$ such that $\lim _{i \rightarrow \infty} p_{c}^{(i)}<p<p_{c}$. Then Lemma 3.2 and Corollary 3.5 ensure that $\forall i \in \mathbb{N}, M^{(i)}(p)>M^{(i)}\left(p_{c}^{(i)}\right) \stackrel{\text { def }}{=} 0$ and Lemma 3.7 that $\mathbb{E}\left[{ }_{p} \bar{X}_{n}^{(i)}\right] \geq n M^{(i)}(p)>$ $0, \forall i \in \mathbb{N}, \forall n \in \mathbb{N}$. Thus

$$
\lim _{i \rightarrow \infty} \mathbb{E}\left[{ }_{p} \bar{X}_{n}^{(i)}\right] \geq 0, \forall n \in \mathbb{N}
$$

(inequality [6] ensures that this limit is well defined)

By the other side,

$$
\begin{array}{rlr}
p<p_{c} & \Longrightarrow \frac{{ }_{p} \bar{X}_{n}^{(\infty)}}{n} \stackrel{(n)}{\longrightarrow}-\infty \text { as } & \text { Lemma 3.1 and commentary on Lemma 3.2 } \\
& \Longrightarrow \mathbb{E}\left[\frac{{ }_{p} \bar{X}_{n}^{\infty}}{n}\right] \stackrel{(n)}{\longrightarrow}-\infty & \text { Fatou's Lemma } \\
& \Longrightarrow \exists \bar{n}: \mathbb{E}\left[{ }_{p} \bar{X}_{\bar{n}}^{\infty}\right]<0 &
\end{array}
$$


Applying Lemma 3.8 for $\bar{n}$ yields

$$
\lim _{i \rightarrow \infty} \mathbb{E}\left[{ }_{p} \bar{X}_{\bar{n}}^{i}\right]=\mathbb{E}\left[{ }_{p} \bar{X}_{\bar{n}}^{\infty}\right]<0
$$

which contradicts inequality 14 above. Hence, $\lim _{i \rightarrow \infty} p_{c}^{(i)} \geq p_{c}$ and the theorem follows from Corollary 3.6

\section{Numerical Calculations}

\subsection{Algebraical Determination of the Critical Probabilities}

Theorem 3.9 (The Convergence Theorem) is of theoretical interest by itself. However weaker results such as Corollaries 3.5 and 3.6 already indicate that each $p_{c}^{(i)}, i \in \mathbb{N}$, is an improved lower bound (regarding its predecessors) for the critical probability of Oriented Percolation.

Although tacitly present in Sections 2.3.3 and 2.4 above, we present below the algorithm for calculating the critical probabilities $p_{c}^{(i)}, i \in \mathbb{N}$ exactly.

According to Definitions 2.2 2.3 2.4 2.5] and 2.6] the critical probabilities $p_{c}^{(i)}, i \in \mathbb{N}$, may be determined in the following steps:

(i) Determination of the Probabilities $P_{(l, m, k)}^{(i)}$ (Def. 2.3) in terms of $q \stackrel{\text { def }}{=} 1-p$ :

elementary combinatorics show that the probabilities $P_{(l, m, k)}^{(i)}, 0 \leq l, m \leq 2^{i}-1, k \in \mathbb{N}$, may be expressed as polinomial functions of $q$, ie

$$
P_{(l, m, k)}^{(i)}=P_{(l, m, k)}^{(i)}(q): \text { polinomial in } q
$$

(ii) Determination of the Transition Matrix $\left(q_{l m}^{(i)}\right)_{0 \leq l, m \leq 2^{i}-1}$ of the IMC ${ }_{p} Y_{\bullet}^{(i)}$ : the transition probabilities defined in (11) may be expressed as

$$
q_{l m}^{(i)}=\sum_{k=0}^{\infty} P_{(l, m, k)}^{(i)}(q)
$$

As the numerical sequence $\left(P_{(l, m, k)}^{(i)}(q)\right)_{k \in \mathbb{N}}$ is a geometric progression (ratio $q^{2}$ ) starting from the $(i+2)^{t h}$ term, the transition probabillities $q_{l m}^{(i)}$ may be expressed as rational functions of $q$. As a matter of fact, these probabilities are always polinomials in $q$ :

$$
q_{l m}^{(i)}=q_{l m}^{(i)}(q): \text { polinomial in } q
$$

(iii) Determination of the Stationary Measure $\left(\pi^{i}\right)$ of the IMC ${ }_{p} Y_{\bullet}^{(i)}$ :

the combinatorial calculus that lead to equation (15) above show that the transition probabilities $q_{l m}^{(i)}(q), 0 \leq l, m \leq 2^{i}-1$, are strictly positive for $q \in(0,1)$, so that the transition matrix $\left(q_{l m}^{(i)}\right)$ is irreducible and aperiodic. Hence $\pi^{i}$ is a well defined probability measure on $\mathcal{S}^{i}$ and can be algebraically determined from $\left(q_{l m}^{(i)}\right)$; resulting that

$$
\pi_{l}^{(i)}(q) \text { : a strictly positive rational function of } q, q \in(0,1), 0 \leq l \leq 2^{i}-1
$$


(iv) Determination of $M_{l}^{(i)}$, the Mean Jump of the SDTCP ${ }_{p} X_{\bullet}^{(i)}$ on State $\sigma_{l}$ :

Definition 2.4 and the fact that the numerical sequence $\left(P_{l m k}^{(i)}(q)\right)_{k \in \mathbb{N}}$ is essentially a geometric progression yield that

$$
M_{l}^{(i)}(q): \text { a rational function of } q, 0 \leq l \leq 2^{i}-1
$$

(v) Determination of $M^{(i)}$, the Mean Jump of the SDTCP ${ }_{p} X_{\bullet}^{(i)}$ :

Definition 2.5 and steps (iii) and (iv) above ensure that

$$
M^{(i)}(q) \text { : a rational function of } q
$$

(vi) Determination of the Critical Probability $p_{c}^{(i)}$ :

according to Corollary 3.3 and Definition 2.6. $p_{c}^{(i)}$ is the only real root of $M^{(i)}(p)$ on the interval $(0,1)$; so that it is the only real root of the polinomial in the numerator of $M^{(i)}(p)$. Its calculation thus can be done numerically.

In the sequel, we employ the algorithm described above for calculating the first critical probability in algebraical terms:

\subsubsection{The Critical Probability of Zero ${ }^{\text {th }}$ Order , $p_{c}^{(0)}$ :}

In this case $\left|\mathcal{S}^{(i)}\right|=1$, so ${ }_{p} Y_{\bullet}^{(i)}$ is trivial:

$$
\begin{aligned}
M^{(0)}(q) & =M_{0}^{(0)}(q)=\sum_{k=0}^{\infty}(1-2 k) \cdot P_{0,0, k}^{(0)}(q)=1 \cdot p-1 \cdot q\left(1-q^{2}\right)-3 \cdot q^{3}\left(1-q^{2}\right)-5 \cdot q^{5}\left(1-q^{2}\right)-\ldots \\
& =(1-q)-q\left(1-q^{2}\right) \cdot\left[1+3 q^{2}+5 q^{4}+7 q^{6}+\ldots\right]=(1-q)-q\left(1-q^{2}\right) \frac{1+q^{2}}{\left(1-q^{2}\right)^{2}}= \\
& =(1-q)-\frac{q+q^{3}}{1-q^{2}}=\frac{1-2 q-q^{2}}{1-q^{2}}
\end{aligned}
$$

Hence,

$$
M^{(0)}(q)=0 \Leftrightarrow 1-2 q-q^{2}=0 \Rightarrow p_{c}^{(0)}=2-\sqrt{2}=0.58579 \ldots
$$

\subsubsection{The Critical Probability of First Order, $p_{c}^{(1)}$ :}

$$
\begin{gathered}
\left(q_{l m}^{(1)}\right)(q)=\left[\begin{array}{cc}
q-q^{3}+q^{4} & 1-q+q^{3}-q^{4} \\
q^{2} & 1-q^{2}
\end{array}\right], \quad \pi_{l}^{(1)}(q)=\frac{\left(q^{2}, 1-q+q^{3}-q^{4}\right)}{1-q+q^{2}+q^{3}-q^{4}} \\
M_{0}^{(1)}(q)=\frac{1-2 q-3 q^{2}+2 q^{4}}{1-q^{2}}, \quad M_{1}^{(1)}(q)=\frac{1-2 q-q^{2}}{1-q^{2}} \\
M^{(1)}(q)=\frac{1-3 q+2 q^{2}-6 q^{4}+q^{5}+3 q^{6}}{1-q+2 q^{3}-2 q^{4}-q^{5}+q^{6}}
\end{gathered}
$$

Hence,

$$
M^{(1)}(q)=0 \Rightarrow p_{c}^{(1)}=0.604233 \ldots
$$




\subsubsection{The Critical Probability of Second Order, $p_{c}^{(2)}$ :}

$$
\begin{aligned}
& \left(q_{l m}^{(2)}(q)\right)=\left[\begin{array}{cccc}
q-q^{3}+q^{6} & q-2 q^{2}+q^{3}+q^{4}-q^{6} & 1-2 q+q^{2}+q^{4}-q^{6} & q^{2}-2 q^{4}+q^{6} \\
q^{2}-q^{3}+q^{4}-q^{5}+q^{6} & q-q^{2}+q^{5}-q^{6} & q-2 q^{2}+2 q^{3}-q^{4}+q^{5}-q^{6} & 1-2 q+2 q^{2}-q^{3}-q^{5}+q^{6} \\
2 q^{3}-q^{4}-q^{5}+q^{6}-q^{7}+q^{8} & 2 q^{2}-3 q^{3}+q^{4}+q^{7}-q^{8} & q-2 q^{3}+2 q^{5}-q^{6}+q^{7}-q^{8} & 1-q-2 q^{2}+3 q^{3}-q^{5}-q^{7}-q^{8} \\
q^{4} & q^{2}-q^{4} & q^{2}-q^{4} & 1-2 q^{2}+q^{4}
\end{array}\right] \\
& \pi_{0}^{(2)}(q)=\frac{-2 q^{4}+2 q^{5}-4 q^{6}+9 q^{7}-14 q^{8}+15 q^{9}-9 q^{10}+2 q^{11}}{-1+3 q-6 q^{2}+8 q^{3}-17 q^{4}+30 q^{5}-44 q^{6}+46 q^{7}-20 q^{8}-17 q^{9}+38 q^{10}-32 q^{11}+13 q^{12}-2 q^{13}} \\
& \pi_{1}^{(2)}(q)=\frac{-q^{2}+2 q^{3}-2 q^{4}+q^{5}-4 q^{6}+9 q^{7}-5 q^{8}-6 q^{9}+12 q^{10}-8 q^{11}+2 q^{12}}{-1+3 q-6 q^{2}+8 q^{3}-17 q^{4}+30 q^{5}-44 q^{6}+46 q^{7}-20 q^{8}-17 q^{9}+38 q^{10}-32 q^{11}+13 q^{12}-2 q^{13}} \\
& \pi_{2}^{(2)}(q)=\frac{-q^{2}+q^{3}+2 q^{5}-9 q^{6}+15 q^{7}-14 q^{8}+8 q^{9}-2 q^{10}}{-1+3 q-6 q^{2}+8 q^{3}-17 q^{4}+30 q^{5}-44 q^{6}+46 q^{7}-20 q^{8}-17 q^{9}+38 q^{10}-32 q^{11}+13 q^{12}-2 q^{13}} \\
& \pi_{3}^{(2)}(q)=\frac{-1+3 q-4 q^{2}+5 q^{3}-13 q^{4}+25 q^{5}-27 q^{6}+13 q^{7}+13 q^{8}-34 q^{9}+37 q^{10}-26 q^{11}+11 q^{12}-2 q^{13}}{-1+3 q-6 q^{2}+8 q^{3}-17 q^{4}+30 q^{5}-44 q^{6}+46 q^{7}-20 q^{8}-17 q^{9}+38 q^{10}-32 q^{11}+13 q^{12}-2 q^{13}} \\
& M_{l}^{(2)}=\frac{1}{1-q^{2}}\left(1-2 q-5 q^{2}+4 q^{4}, 1-2 q-3 q^{2}+2 q^{4}, 1-2 q-q^{2}-2 q^{4}+2 q^{6}, 1-2 q-q^{2}\right) \\
& M^{(2)}(q)=\frac{-1+5 q-11 q^{2}+16 q^{3}-25 q^{4}+52 q^{5}-75 q^{6}+96 q^{7}-58 q^{8}-69 q^{9}+152 q^{10}-111 q^{11}-5 q^{12}+74 q^{13}-49 q^{14}+10 q^{15}}{\left(1-q^{2}\right)\left(-1+3 q-6 q^{2}+8 q^{3}-17 q^{4}+30 q^{5}-44 q^{6}+46 q^{7}-28 q^{8}-17 q^{9}+38 q^{10}-32 q^{11}+13^{12}-2 q^{13}\right)}
\end{aligned}
$$

Hence,

$$
M^{(2)}(q)=0 \Rightarrow p_{c}^{(2)}=0.614187
$$

\subsection{Numerical Determination of the Critical Probabilities:}

The combinatorial calculations leading to the transition matrix $\left(q^{(i)}\right)_{l m}$ and to the mean-drift vector $M(i)_{l}$ described in Section 4.1 above, yet fastidious for humans, are taylor-made for computers: while it took us a whole afternoom for calculating $\left(q^{(2)}\right)_{l m}$ algebraically (16 polinomial entries), a FORTRAN 77 program, running at $600 \mathrm{MHz}$, calculated $\left(q^{(9)}\right)_{l m}\left(4^{9}\right.$ polinomial entries $)$ in less than one minute time!

The greatest problem in writing a computer program version for the algorithm described in Section 4.1 arises precisely in step(iii), viz. the determination of the stationary measure $\pi^{(i)}$ (in algebraical terms), starting from the transition matrix $\left(q^{(i)}\right)_{l m}(q)$. True, it can be done straightforwardly, acting on the polinomials as if they were numbers. However, if we don't take into account the eventual and probable simplifications that may take place, the degree of the outcoming polinomials soon get unmanageble. And finding out these simplifications is far from obvious.

Therefore we have adopted a different approach:

(i) After having algebraically calculated the transition matrix $\left(q^{(i)}\right)_{l m}(q)$ and the mean-drift vector $M_{l}^{(i)}(q)$, the program calculated a numerical transition matrix and a numerical mean-drift vector for a decreasing sequence of numerical values of $q$.

(ii) From each numerical transition matrix, a numerical stationary measure was obtained by solving a system of linear equations. It is worth-while mentioning that partial pivoting [5] was of fundamental importance in keeping numerical errors under controll.

(iii) The numerical mean-drift was then obtained, performing the inner-product of Definition 2.5]

(iv) According to Definition $\left[2.6 q_{c}^{(i)}\left(\stackrel{\text { def }}{=} 1-p_{c}^{(i)}\right)\right.$ lies between the last value of $q$ for which $M^{(i)}(q)$ is negative and the first value of $q$ for which $M^{(i)}(q)$ is positive. 
This approach has the onus of introducing numerical erros (basically in step ii above), from which we hitherto have got rid of. So as to produce reliable numerical data for the critical probabilities, rigorous upper bounds for the numerical errors must be provided. In doing so, we followed the set-up of foward analysis described in [5], which consists fundamentally in providing partial upper bounds after each arithmetic operation performed in the flow of numerical calculations leading to the final numerical result. This rather crude approach has the advantage of providing a secure upper bound for numerical errors, regardless of particular features the linear system may present, as it is often the case in the so called backward analysis ( [5]).

In the sequel, we present the numerical data produced following steps i-iv above ${ }^{6}$ :

\begin{tabular}{|c|c|c|}
\multicolumn{3}{|c}{$\mathrm{i}=1$} \\
\hline $\mathrm{p}$ & $M_{\text {Num }}^{(1)}(p)$ & $\Delta M^{(1)}(p)$ \\
\hline 0.604231 & $-9.15 \mathrm{E}-6$ & $6.2 \mathrm{E}-16$ \\
0.604232 & $-5.27 \mathrm{E}-6$ & $6.2 \mathrm{E}-16$ \\
0.604233 & $-1.40 \mathrm{E}-6$ & $6.2 \mathrm{E}-16$ \\
0.604234 & $+2.47 \mathrm{E}-6$ & $6.2 \mathrm{E}-16$ \\
\hline
\end{tabular}

\begin{tabular}{|c|c|c|}
\hline \multicolumn{3}{c}{$\mathrm{i}=2$} \\
$\mathrm{p}$ & $M_{\text {Num }}^{(2)}(p)$ & $\Delta M^{(2)}(p)$ \\
\hline 0.614185 & $-1.01 \mathrm{E}-5$ & $6.1 \mathrm{E}-15$ \\
0.614186 & $-5.85 \mathrm{E}-6$ & $6.2 \mathrm{E}-15$ \\
0.614187 & $-1.57 \mathrm{E}-6$ & $6.1 \mathrm{E}-15$ \\
0.614188 & $+2.71 \mathrm{E}-6$ & $6.2 \mathrm{E}-15$ \\
\hline
\end{tabular}

\begin{tabular}{|c|c|c|}
\hline \multicolumn{3}{c}{$\mathrm{i}=3$} \\
$\mathrm{p}$ & $M_{\text {Num }}^{(3)}(p)$ & $\Delta M^{(3)}(p)$ \\
\hline 0.620203 & $-1.36 \mathrm{E}-5$ & $2.5 \mathrm{E}-14$ \\
0.620204 & $-8.96 \mathrm{E}-6$ & $2.5 \mathrm{E}-14$ \\
0.620205 & $-4.32 \mathrm{E}-6$ & $2.6 \mathrm{E}-14$ \\
0.620206 & $+3.15 \mathrm{E}-7$ & $2.6 \mathrm{E}-14$ \\
\hline
\end{tabular}

\begin{tabular}{|c|c|c|}
\hline $\mathrm{p}$ & $M_{N u m}^{(4)}(p)$ & $\Delta M^{(4)}(p)$ \\
\hline 0.624210 & $-9.52 \mathrm{E}-6$ & $8.0 \mathrm{E}-14$ \\
0.624211 & $-4.57 \mathrm{E}-6$ & $8.0 \mathrm{E}-14$ \\
0.624212 & $+3.71 \mathrm{E}-7$ & $8.0 \mathrm{E}-14$ \\
& & \\
\hline
\end{tabular}

\begin{tabular}{|c|c|c|}
\hline \multicolumn{3}{|c}{$\mathrm{i}=5$} \\
$\mathrm{p}$ & $M_{N u m}^{(5)}(p)$ & $\Delta M^{(5)}(p)$ \\
\hline 0.627064 & $-1.31 \mathrm{E}-5$ & $1.9 \mathrm{E}-13$ \\
0.627065 & $-7.85 \mathrm{E}-6$ & $1.9 \mathrm{E}-13$ \\
0.627066 & $-2.63 \mathrm{E}-6$ & $1.9 \mathrm{E}-13$ \\
0.627067 & $+2.60 \mathrm{E}-6$ & $1.9 \mathrm{E}-13$ \\
\hline
\end{tabular}

\begin{tabular}{|c|c|c|}
\hline $\mathrm{p}$ & $\mathrm{i}=6$ \\
\hline 0.629201 & $-1.55 \mathrm{E}-5$ & $3.8 \mathrm{E}-13$ \\
0.629202 & $-9.98 \mathrm{E}-6$ & $3.8 \mathrm{E}-13$ \\
0.629203 & $-4.51 \mathrm{E}-6$ & $3.9 \mathrm{E}-13$ \\
0.629204 & $+9.66 \mathrm{E}-7$ & $3.8 \mathrm{E}-13$ \\
\hline
\end{tabular}

\begin{tabular}{|c|c|c|}
\multicolumn{3}{c}{$\mathrm{i}=7$} \\
$\mathrm{p}$ & $M_{\text {Num }}^{(7)}(p)$ & $\Delta M^{(7)}(p)$ \\
\hline 0.630863 & $-1.01 \mathrm{E}-5$ & $7.2 \mathrm{E}-13$ \\
0.630864 & $-4.41 \mathrm{E}-6$ & $7.2 \mathrm{E}-13$ \\
0.630865 & $+1.30 \mathrm{E}-6$ & $7.2 \mathrm{E}-13$ \\
& & \\
\hline
\end{tabular}

\begin{tabular}{|c|c|c|}
\multicolumn{3}{c}{$\mathrm{i}=8$} \\
$\mathrm{p}$ & $M_{\text {Num }}^{(8)}(p)$ & $\Delta M^{(8)}(p)$ \\
\hline 0.632192 & $-8.53 \mathrm{E}-6$ & $1.3 \mathrm{E}-12$ \\
0.632193 & $-2.60 \mathrm{E}-6$ & $1.2 \mathrm{E}-12$ \\
0.632194 & $+3.32 \mathrm{E}-6$ & $1.3 \mathrm{E}-12$ \\
& & \\
\hline
\end{tabular}

\begin{tabular}{|c|c|c|}
\hline $\mathrm{i}=9$ \\
\hline $\mathrm{p}$ & $M_{\text {Num }}^{(9)}(p)$ & $\Delta M^{(9)}(p)$ \\
\hline 0.63326 & $-1.29 \mathrm{E}-4$ & $2.0 \mathrm{E}-12$ \\
0.63327 & $-6.79 \mathrm{E}-5$ & $2.0 \mathrm{E}-12$ \\
0.63328 & $-6.68 \mathrm{E}-6$ & $2.0 \mathrm{E}-12$ \\
0.63329 & $+5.45 \mathrm{E}-5$ & $2.0 \mathrm{E}-12$ \\
\hline
\end{tabular}

Based upon the data displayed above, the first ten critical probabilities may be determined:

\begin{tabular}{|c||c|c|c|c|c|c|c|c|c|c|}
\hline$i$ & 0 & 1 & 2 & 3 & 4 & 5 & 6 & 7 & 8 & 9 \\
\hline$p_{c}^{(i)}$ & 0.585787 & 0.604233 & 0.614187 & 0.620205 & 0.624211 & 0.627066 & 0.629203 & 0.630864 & 0.632193 & 0.63328 \\
\hline
\end{tabular}

So that we can establish the inequality : $\quad p_{c} \geq 0.63328$

\footnotetext{
${ }^{6}$ the calculations were carried out on double precision.

${ }^{7}\left|M^{(i)}(p)-M_{N u m}^{(i)}(p)\right| \leq \Delta M^{(i)}(p) ;$ where $M_{N u m}^{(i)}(p)$ denotes the numerically calculated mean drift of $i^{\text {th }}$ order.
} 


\section{Simulations}

The boolean and inductive features of definition [2.1 make it extremely suitable for computer simulations: at each step a FORTRAN77-written computer program determmines the value of ${ }_{p} \bar{X}_{n}^{(i)} / n$, the right edge mean speed at moment $n$. In accordance with lemma [3.1 this sequence converges $(a . s$. in $n)$ to $M^{(i)}(p)$, the right edge mean speed. Therefore running the program for different values of $p$, one can estimate the value of $p_{c}^{(i)}$ observing the height of the plateau established with the increase of $n$, according with the rule:

(a) height of plateau $<0 \Rightarrow p<p_{c}^{(i)}$,

(b) height of plateau $>0 \Rightarrow p>p_{c}^{(i)}$.

The two figures displayed below ilustrate the use of this technique when $i=1000$ :

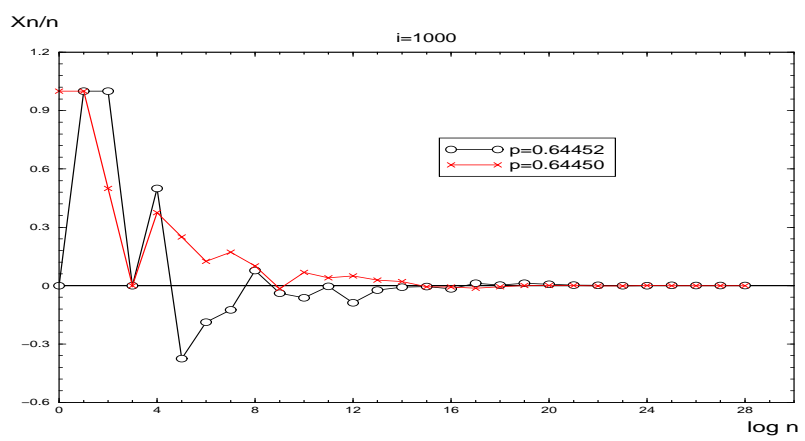

Figure 4: Independent (not coupled) trajectories of the processes ${ }_{.64450} \bar{X}_{n}^{(1000)} / n$ e ${ }_{.64452} \bar{X}_{n}^{(1000)} / n$.

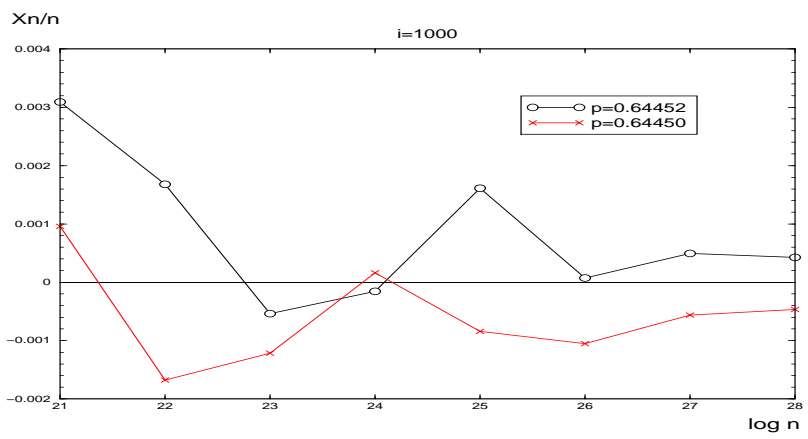

Figure 5: Enlarged detail of the figure above . 
Table @below sumarizes the numerical results obtained by means of the technique for different values of $i$ :

\begin{tabular}{||c|c||}
\hline \hline$i$ & $p_{c}^{(i)}$ \\
\hline 5 & 0,627 \\
6 & 0,629 \\
9 & 0,6332 \\
20 & 0,638 \\
40 & 0,641 \\
100 & 0,643 \\
200 & 0,6438 \\
1000 & 0,64451 \\
\hline \hline
\end{tabular}

Table 1: Critical probabilities obtained by means of simulation.

It is interesting to observe that the plateau pattern exhibited in figure 4 is present even in the vicinity of criticality, ie $p \approx p_{c}^{(i)}$ and $i \nearrow \infty$. Therefore the critical probabilities $p_{c}^{(i)}$ (even for large values of $i$ and so very close to criticality) can be estimated within the desired precision simply by increasing the value of $n$, ie running the program until the plateau pattern becomes clear. We belive that this feature distinguishes our technique from the usual Monte Carlo simulation methods, wherein instability appears near criticality and the use of scaling techniques is called for.

\section{Acknowledgement:}

The authors are indebted to M. Menshikov, who first suggested the use of Markov chains in a strip to track the edge of Oriented Percolation...

\section{References}

[1] R. Durrett. (1984) Oriented Percolation in Two Dimensions.

The Annals of Probability, 12(4), 999-1040.

[2] G. Grimmett. (1999) Percolation. Springer Verlag.

[3] G.Fayolle/V.A.Malyshev/M.V.Menshikov. (1995)

Topics in the Constructive Theory of Countable Markov Chains. Cambridge University Press .

[4] R. G. Bartle. (1995)

The Elements of Integration and Lebesgue Measure. John Willey \& Sons Inc.

[5] J. H. Wilkinson. (1963) Rounding Errors in Algebraic Processes. Her Majesty's Stationary Office. 